\title{
Elimination of proinflammatory cytokines in pediatric cardiac surgery: Analysis of ultrafiltration method and filter type
}

\author{
Pascal A. Berdat, MD \\ Evelyne Eichenberger ${ }^{a}$ \\ Julia Ebell ${ }^{\mathrm{a}}$ \\ Jean-Pierre Pfammatter, MD \\ Mladen Pavlovic, $\mathrm{MD}^{\mathrm{b}}$ \\ Claudia Zobrist, $\mathrm{MD}^{\mathrm{c}}$ \\ Erich Gygax ${ }^{a}$ \\ Urs Nydegger, $\mathrm{MD}^{\mathrm{a}}$ \\ Thierry Carrel, MDa
}

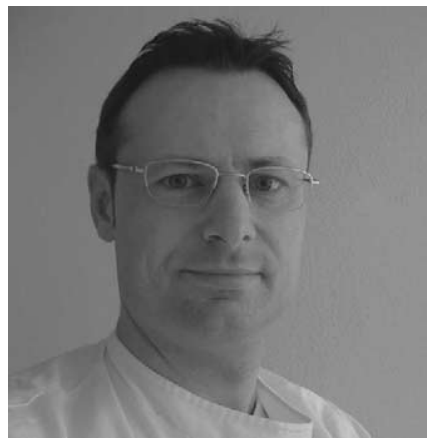

Dr Berdat

From the Clinic for Cardiovascular Surgery, ${ }^{\text {a }}$ the Division of Pediatric Cardiology, ${ }^{b}$ and the Division of Cardiovascular Anesthesiology, ${ }^{c}$ University Hospital, Berne, Switzerland

Received for publication April 30, 2003; revisions requested Nov 27, 2003; accepted for publication Jan 21, 2004.

Address for reprints: Pascal A. Berdat, MD, Clinic for Cardiovascular Surgery, Swiss Cardiovascular Center Berne, University Hospital, CH-3010 Berne, Switzerland (Email: pascal.berdat@insel.ch).

J Thorac Cardiovasc Surg 2004;127: 1688-96

$0022-5223 / \$ 30.00$

Copyright (C) 2004 by The American Association for Thoracic Surgery

doi:10.1016/j.jtcvs.2004.01.030
Objective: This study was undertaken to assess whether different filter types or ultrafiltration methods influence inflammatory markers in pediatric cardiac surgery.

Methods: Forty-one children younger than 5 years were prospectively randomized to groups A (polyamid filter with conventional ultrafiltration), B (polyamid filter with modified ultrafiltration), C (polysulfon filter with conventional ultrafiltration), and D (polysulfon filter with modified ultrafiltration). Interleukin 6 , interleukin 10, tumor necrosis factor, terminal complement complex, and lactoferrin were measured before the operation (T0), before rewarming (T1), after ultrafiltration (T2), at 6 (T3) and 18 hours (T4) after the operation, and in the ultrafiltrate.

Results: All markers changed with both ultrafiltration methods, both filter types, and in all groups (except tumor necrosis factor) along the T0 to T4 observation time ( $P$ $<$.0001). Their patterns of changes were different for terminal complement complex, with less decrease after use of the polysulfon filter $(P<.05)$, and among groups A through $\mathrm{D}$ for interleukin $6(P=.01)$, with more decrease in group $\mathrm{C}$ than group A $(P<.02)$. Interleukin 10 decreased with the polyamid filter $(P<.001)$ but not with the polysulfon filter. In the ultrafiltrate, tumor necrosis factor was higher with the polysulfon filter than the polyamid filter $(6.8 \pm 5 \mathrm{pg} / \mathrm{mL}$ vs $4.0 \pm 3.7$ $\mathrm{pg} / \mathrm{mL}, P<.05)$. The ultrafiltrate/plasma ratio of interleukin 6 was higher with conventional ultrafiltration than modified ultrafiltration $(0.018 \pm 0.017$ vs $0.004 \pm$ $0.007, P<.005)$.

Conclusions: The polysulfon filter showed a filtration profile for inflammatory mediators superior to that of the polyamid filter for interleukin 6 , tumor necrosis factor, and interleukin 10. Interleukin 6 was most efficiently removed by conventional ultrafiltration with a polysulfon filter, and tumor necrosis factor was best removed by modified ultrafiltration with a polysulfon filter, whereas other inflammatory mediators were not influenced by filter type or ultrafiltration method. Therefore combined conventional and modified ultrafiltration with a polysulfon filter may currently be the most effective strategy for removing inflammatory mediators in pediatric heart surgery. 


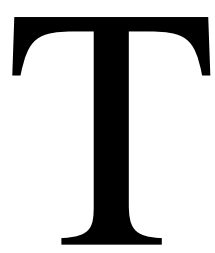

he proinflammatory response to cardiac surgery, induced by the production of cytokines triggered by cardiopulmonary bypass (CPB) ${ }^{1-3}$ contributes significantly to postoperative morbidity and mortality ${ }^{4}$ and is accentuated in pediatric patients. ${ }^{5,6}$ With ultrafiltration, most of the excess free water and a part of these mediators may be eliminated, and the inflammatory response to $\mathrm{CPB}$ thus may be attenuated. ${ }^{4,7}$ Conventional ultrafiltration (CUF), applied during the rewarming phase on CPB, and modified ultrafiltration (MUF), performed immediately after cessation of $\mathrm{CPB}$, are both broadly used in pediatric cardiac surgery. ${ }^{8-11}$ However, conflicting data exist concerning their efficacy in removing cytokines, ${ }^{4-6,9,12-15}$ and controversy has mainly focused on differences in ultrafiltration methods but not in filter performance. Additionally, comparison of results from ultrafiltration studies are difficult because of variations in ultrafiltration techniques, equipment, definitions of study end points, and cytokine measurements. Since 1973 different types of hemofilters, mainly polycarbon, have been used to eliminate the excess water from the priming volume according to the pressure gradient principle. These filters have permanently been improved, replaced by polyarylethersulfon filters (PSF) in 1986 and later by the most recent filter generation, the polyamid filter (PAF). PSF does not have ideal filtration properties, because high pressure gradients are needed and filtration rates can be controlled only approximately. The improved biocompatibility of the PAF, along with its reduced surface, effectiveness of filtration under physiologic pressure, and excellent handling properties, makes its use for ultrafiltration attractive. The efficacy of these filters in removing inflammatory mediators, simultaneously taking into consideration different ultrafiltration methods, has not however been investigated in a direct comparative fashion so far. To optimize efficacy of ultrafiltration, methods and filter types need be harmonized, combining a particular ultrafiltration method with a specific filter type, to allow more efficient elimination of cytokines. The aim of this study was to assess the effectiveness of PSF and PAF filters concerning elimination of cytokines in relation to the ultrafiltration method used. Secondarily, we wanted to define an optimal combination of filter type and ultrafiltration method in pediatric cardiac surgery.

\section{Patients and Methods}

\section{Patients}

Forty-one pediatric patients scheduled for elective cardiac surgery with the use of CPB were included in the study. Children 5 years or older, those undergoing surgical procedures without $\mathrm{CPB}$ or with $\mathrm{CPB}$ duration of less than 45 minutes, and those undergoing emergency procedures were excluded from the study. Patients were prospectively randomly assigned to 4 groups for type of filter (PAF vs PSF) and mode of ultrafiltration (CUF vs MUF): group A, PAF with CUF; group B, PAF with MUF; group C, PSF with CUF, and group D, PSF with MUF. The study protocol was approved by the local ethical committee. Informed consent was obtained from the parents of each child. Patients characteristics and operative data are depicted in Table 1. Surgery consisted of ventricular septal defect closure $(n=15)$; repair of tetralogy of Fallot or doubleoutlet right ventricle $(\mathrm{n}=6)$; complete atrioventricular canal repair $(n=4)$; Rastelli operation $(n=2)$; patch enlargement plasty of the ascending aorta, aortic arch, or pulmonary truncus $(\mathrm{n}=5)$; arterial switch operation $(\mathrm{n}=2)$; tetralogy of Fallot reoperations $(n=2)$; and miscellaneous other procedures $(n=5)$. No significant differences were observed between the groups with respect to patients' diagnoses and demographic data.

\section{Surgical Technique}

In all cases the surgical approach was through a median sternotomy with CPB and systemic hypothermia. Two patients were operated on with a period of deep hypothermic circulatory arrest. Cold antegrade blood cardioplegia without topical cooling and warm reperfusion cardioplegia (hot shot) were routinely used. Non-heparin-coated CPB circuits of different sizes were used, with a Polystan Safe Mini oxygenator (Polystan, Vaerlose, Denmark) for children smaller than $5 \mathrm{~kg}$ and a Dideco Midiflow oxygenator (Dideco, Mirandola, Italy) for children larger than 5 kg. Aprotinin (Trasylol; Bayer AG, Leverkusen, Germany) at a dosage of $300,000 \mathrm{kIU} / 1000 \mathrm{~mL}$ circulating volume (priming plus blood volume) was added to the blood prime in all patients. Heparin was given to achieve an activated clotting time greater than 600 seconds. After cessation of CPB, protamine sulfate (Protamin; Bayer AG) was administered to reestablish preoperative activated clotting time. Mediastinal shed blood was not retransfused.

\section{Anesthesia}

All patients were given midazolam (Dormicum; Roche Pharma AG, Reinach, Switzerland) at 0.5 to $1 \mathrm{mg} / \mathrm{kg}$ rectally for premedication. Anesthesia was induced with thiopental (Pentothal; Abbott AG, Baar, Switzerland) with midazolam or ketamine (Ketalar; Pfizer AG, Zurich, Switzerland) with midazolam and continued with high-dose fentanyl (Fentanyl-Jansson; Janssen-Cilag AG, Baar, Switzerland) and isoflurane (Forene; Abbott AG) or ketamine infusion of $2 \mathrm{mg} / \mathrm{kg}$. Muscle relaxation was achieved with pancuronium (Pavulon; Organon AG, Pfäffikon, Switzerland). Patients were mechanically ventilated with mixed air and oxygen (40\%-50\%) and extubated in the intensive care unit. Hemodynamics were routinely assessed by transesophageal echocardiography and surgical introduction of a pulmonary artery pressure catheter. For postoperative pain relief, patients were given morphine (Morphin-HCl; Sintetica SA, Mendrisio, Switzerland) supplemented with acetaminophen (INN: paracetamol, Dafalgan; UPSAmedica $\mathrm{GmbH}$, Baar, Switzerland). Patients received antibiotic prophylaxis with intravenous cefuroxime at $50 \mathrm{mg} / \mathrm{kg}$ and continued at $100 \mathrm{mg} /(\mathrm{kg} \cdot \mathrm{d})$ (Zinacef; GlaxoSmithKline AG, Münchenbuchsee, Switzerland) for at least 24 hours until all drains were removed.

\section{Blood Sample Protocol}

Blood samples for analysis of cytokine concentrations were collected from the radial artery after induction of anesthesia (T0), before rewarming (T1), after ultrafiltration (T2), at 6 (T3) and 18 hours (T4) after the operation. Blood was collected into sterile ethylenediaminetetraacetic acid and serum tubes and immediately 
TABLE 1. Patients characteristics and operative data

\begin{tabular}{|c|c|c|c|c|}
\hline Variable & $\begin{array}{c}A \\
(n=11)\end{array}$ & $\begin{array}{c}B \\
(n=11)\end{array}$ & $\begin{array}{c}C \\
(n=10)\end{array}$ & $\begin{array}{c}D \\
(n=9)\end{array}$ \\
\hline Age (y) & $1.4 \pm 1.4$ & $2.5 \pm 1.9$ & $1.3 \pm 1.3$ & $1.6 \pm 1.6$ \\
\hline Body surface area $\left(\mathrm{m}^{2}\right)$ & $0.406 \pm 0.13$ & $0.5 \pm 0.18$ & $0.39 \pm 0.15$ & $0.42 \pm 0.15$ \\
\hline Operation time (min) & $188.2 \pm 55$ & $173.6 \pm 45$ & $185.6 \pm 85$ & $198.9 \pm 43$ \\
\hline CPB time (min) & $96.8 \pm 30$ & $97.2 \pm 41$ & $106 \pm 43$ & $95 \pm 26$ \\
\hline Aortic crossclamp time (min) & $50.7 \pm 22$ & $54.8 \pm 31$ & $56.1 \pm 23$ & $42.3 \pm 21$ \\
\hline Lowest body temperature $\left({ }^{\circ} \mathrm{C}\right)$ & $28.8 \pm 5.1$ & $31.5 \pm 2.2$ & $28.4 \pm 4.7$ & $30.3 \pm 4.4$ \\
\hline Cardioplegia volume $(\mathrm{mL} / \mathrm{kg})$ & $31.6 \pm 20.4$ & $32.6 \pm 26.5$ & $51 \pm 37.3$ & $183 \pm 165$ \\
\hline Ultrafiltration time (min) & $28.5 \pm 11$ & $22.8 \pm 10$ & $25.5 \pm 10$ & $27.7 \pm 33$ \\
\hline Ultrafiltration volume $(\mathrm{mL} / \mathrm{kg})$ & $115 \pm 66$ & $82.3 \pm 45$ & $111.7 \pm 62$ & $96.7 \pm 55$ \\
\hline Ultrafiltration rate maximum (mL/min) & $72 \pm 79$ & $94 \pm 88$ & $45 \pm 60$ & $102 \pm 77$ \\
\hline Ultrafiltration rate minimum (mL/min) & $62 \pm 72$ & $82 \pm 72$ & $42 \pm 58$ & $93 \pm 76$ \\
\hline Filter pressure gradient $(\mathrm{mm} \mathrm{Hg})$ & $70 \pm 41$ & $76 \pm 47$ & $77 \pm 32$ & $61 \pm 38$ \\
\hline $\begin{array}{l}\text { Hematocrit increase during } \\
\text { ultrafiltration }(\%)\end{array}$ & $5.6 \pm 4.4$ & $7.6 \pm 4.6$ & $3.9 \pm 4.8^{*}$ & $8.9 \pm 3.3$ \\
\hline Total blood loss $(\mathrm{mL} / \mathrm{kg})$ & $25 \pm 13$ & $32 \pm 23$ & $25 \pm 16$ & $20 \pm 10$ \\
\hline $\mathrm{RBC}$ transfusion intraoperative $(\mathrm{mL} / \mathrm{kg})$ & $111 \pm 104$ & $73 \pm 48$ & $119 \pm 70$ & $122 \pm 55$ \\
\hline $\mathrm{RBC}$ transfusion postoperative $(\mathrm{mL} / \mathrm{kg})$ & $16 \pm 14$ & $18 \pm 17$ & $11 \pm 9$ & $6 \pm 9$ \\
\hline $\mathrm{RBC}$ transfusion total $(\mathrm{mL} / \mathrm{kg})$ & $127 \pm 105$ & $91 \pm 49$ & $130 \pm 69$ & $128 \pm 57$ \\
\hline FFP transfusion intraoperative $(\mathrm{mL} / \mathrm{kg})$ & $13 \pm 24^{*}$ & $21 \pm 24$ & $16 \pm 19$ & $42 \pm 37$ \\
\hline FFP transfusion postoperative $(\mathrm{mL} / \mathrm{kg})$ & $3 \pm 5 \dagger$ & $9 \pm 8$ & $12 \pm 12$ & $11 \pm 15$ \\
\hline FFP transfusion total $(\mathrm{mL} / \mathrm{kg})$ & $16 \pm 24^{*}$ & $31 \pm 29$ & $28 \pm 21$ & $54 \pm 43$ \\
\hline
\end{tabular}

All values are mean $\pm \mathrm{SD}$. $R B C$, Red blood cell concentrate; FFP, fresh-frozen plasma.

${ }^{*} P<.02$ versus group $D$.

$\mathrm{t} P<.05$ versus groups $\mathrm{B}$ and $\mathrm{C}$.

centrifuged at $2000 \mathrm{~g}$ for 15 minutes at $6^{\circ} \mathrm{C}$. Plasma, ultrafiltrate, and urine samples were stored in multiple aliquots at $-70^{\circ} \mathrm{C}$ until analysis and were thawed only once. At each time point the hematocrit was measured.

\section{Ultrafiltration Technique}

CUF was started with rewarming until cessation of CPB and was stopped after complete extraction of the priming volume. During MUF, the aortic cannula was used to drain the blood across the filter and one atrial cannula was used to return the filtered blood without an auxiliary blood pump. Thus MUF was passively driven by the gradient between aorta and right atrium and assisted by application of vacuum (approximately $20 \mathrm{~cm} \mathrm{H}_{2} \mathrm{O}$ ) at the ultrafiltrate outlet port of the filter. CPB was bypassed by this ultrafiltration circuit, and therefore only the patient volume was filterable. MUF was started after cessation of CPB, as soon as the child was hemodynamically stable, and performed for a period of 20 minutes. During aortic crossclamping, hemofiltration was performed to eliminate $50 \%$ of the priming volume, and during CUF or MUF, the residual $50 \%$ was eliminated. The volume filtered was replaced 1:1 with packed red blood cells until a hematocrit of $30 \%$ was reached; thereafter, it was replaced with fresh-frozen plasma. In groups A and B, a PAF (Jostra BC20 Blood Concentrator filter; Jostra Medizintechnik AG, Hirrlingen, Germany) with a surface of $0.2 \mathrm{~m}^{2}$, a priming volume of $13 \mathrm{~mL}$, a maximal transmembranous gradient of $600 \mathrm{~mm} \mathrm{Hg}$, a luminal diameter of $22 \mu \mathrm{m}$, and a membrane thickness of $50 \mu \mathrm{m}$ was used. In groups C and D, a PSF (Jostra BC60 Blood Concentrator filter; Jostra Medizintechnik AG) with a surface of $0.65 \mathrm{~m}^{2}$, a priming volume of $55 \mathrm{~mL}$, a maximal transmembranous gradient of $600 \mathrm{~mm} \mathrm{Hg}$, a luminal diameter of $215 \mu \mathrm{m}$, and a membrane thickness of $50 \mu \mathrm{m}$ was used.

\section{Cytokine and Complement Analyses}

Interleukin (IL) 6, IL-10, and tumor necrosis factor $\alpha$ (TNF) were measured with specific enzyme immunoassays (Pierce Endogen, Rockford, Ill). To estimate activation of the complement system, we chose the terminal soluble C5b-9 complement complex (TCC) to reflect effector consequence of all three activation pathways (classical, alternative, and mannose-binding protein). TCC was also measured by enzyme immunoassay (Quidel, Mountain View, Calif), as was lactoferrin (Oxis International, Inc, Portland, Ore). All enzyme immunoassays were performed according to the recommendations of the manufacturers. All cytokine concentrations (except those of the ultrafiltrate) were corrected for the actual hematocrit to correct for the high variability of hemodilution. Values measured below the detection limit of a specific test were set equal to the lower limit for statistical analysis.

\section{Statistics}

Data are expressed as mean $\pm \mathrm{SD}$. Percentages are given where appropriate. Statistical analysis was performed with Statview 5.0.1 for Windows (SAS Institute Inc, Cary, NC). For continuous variables comparing serial values, repeated-measures analysis of variance was used. For within-group and between-group analyses, a Mann-Whitney $U$ test and a Fisher exact test were used, respectively. Bonferroni correction was applied where appropriate.

\section{Results}

Intraoperative data were not different among the groups (Table 1). In fact, CUF did not prolong CPB and MUF did not prolong operation time. Total MUF time was significantly longer than total CUF time (defined as the sum of 
hemofiltration time during aortic crossclamp and MUF or CUF time, $45.25 \pm 15.1$ minutes vs $36.6 \pm 11.5$ minutes, $P$ $<.05$ ). However, net CUF time was similar to net MUF time (defined as CUF or MUF time without hemofiltration time, $27 \pm 10.5$ minutes vs $23.5 \pm 9.5$ minutes, $P>.05$ ) without significant differences among the groups. Ultrafiltration volumes, maximal and minimal ultrafiltration rates, and pressure gradients were not significantly different among the groups. Hematocrit increased in all groups during ultrafiltration, demonstrating the efficacy of the ultrafiltration to hemoconcentrate. The increase in hematocrit was higher with MUF than CUF, reaching statistical significance for group D vs group $\mathrm{C}(P<.01$; Table 1$)$.

\section{Inflammatory Markers}

All inflammatory markers except TNF showed a marked increase during surgery (IL-6, IL-10, TCC) or in the first 6 hours thereafter (IL-6, lactoferrin), with return to baseline levels within 18 hours except for lactoferrin.

Repeated-measures analysis of variance (Figure 1, $A-E$ ) revealed no significant effect of the ultrafiltration method (CUF vs MUF) on IL-6, IL-10, TNF, lactoferrin, and TCC. Plasma concentrations of all inflammatory markers changed significantly with both ultrafiltration methods along the T0 to T4 observation time $(P<.0001)$, but the patterns of changes of all markers were not different between the ultrafiltration methods. Testing the effect of the filter type (PAF vs PSF) showed no significant difference regarding IL-6, IL-10, TNF, and lactoferrin. Plasma concentrations of all inflammatory markers changed significantly with both filter types along the T0 to T4 observation time $(P<.0001)$, but the patterns of changes of all markers were not different between the filter types. With the use of PSF, TCC showed a significantly different changing pattern than with $\mathrm{PAF}(P<$ .05 ), with less decrease after filtration (Figure 1, $D$, Table 2). Testing the effect of the 4 combinations of filter type and ultrafiltration method on IL-6, IL-10, TNF, lactoferrin, and TCC revealed no significant differences among groups A through D. All markers except TNF changed significantly along the T0 to T4 observation time $(P<.0001)$. Only the patterns of changes of IL-6 (Figure 1, A) were significantly different among groups A through $\mathrm{D}(P=.01)$, especially between $\mathrm{A}$ and $\mathrm{C}(P<.02)$.

In analyzing the filtration (T1-T2) and immediate postfiltration (T2-T3) periods, significant differences were detected. During filtration, IL-6 increased significantly in groups A $(P<.005)$ and $\mathrm{B}(P<.005$; Figure $1, A)$, with both filter types (PAF $P<.0001$, PSF $P<.0002$ ) and both ultrafiltration methods (CUF $P<.0001$, MUF $P=.0001$; Table 3). IL-10 increased significantly only with the use of PAF $(P=.0002$; Figure $1, B)$ but with either ultrafiltration method (CUF $P<.001$, MUF $P<.005$; Table 3); not, however, in groups A through D. TNF, lactoferrin, and TCC did not change significantly among groups A through $\mathrm{D}$, nor did they change with filter types or ultrafiltration methods (Tables 2 and 3). At T2, IL-6 was significantly lower in group $C$ than in group D $(352.5 \pm 678.2$ vs $1464.0 \pm 855.8$ $\mathrm{pg} / \mathrm{mL}, P<.01)$ and lower after CUF than after MUF $(P<$ .03). After filtration (T2-T3), IL-6 decreased significantly after the use of either filter type (PAF $P=.0002$, PSF $P=$ $.005)$ but only after MUF $(P=.0002)$, not after CUF or within groups A through D. IL-10 decreased significantly only after the use of PAF $(P<.001)$ and with either ultrafiltration method (CUF $P<.01$, MUF $P<.01$ ). TNF decreased significantly only after MUF $(P<.002$; Figure 1 , $C)$ and TCC only after the use of PAF $(P<.0005$; Figure $1, D)$ and CUF $(P<.005)$. In contrast, lactoferrin increased significantly in group A $(P<.005$; Figure $1, E)$, after use of either filter type (PAF $P=.0005$, PSF $P<.005)$ and after CUF $(P<.0001)$.

\section{Ultrafiltrate}

IL-6, IL-10, lactoferrin, and TCC concentrations in the ultrafiltrate were not different among the groups (Table 4). Lactoferrin levels were all below detection limit in all groups. TNF concentrations were significantly higher after the use of PSF than PAF $(6.81 \pm 5.03 \mathrm{pg} / \mathrm{mL}$ vs $4.02 \pm$ $3.67 \mathrm{pg} / \mathrm{mL}, P<.05)$ and higher in group $\mathrm{D}$ than in group A $(7.06 \pm 4.39 \mathrm{pg} / \mathrm{mL}$ vs $2.82 \pm 2 \mathrm{pg} / \mathrm{mL}, P<.082$; Figure $2, A)$. Additionally, the total amount of TNF was significantly higher in group D than in group A (4750 \pm 2593.5 $\mathrm{pg} / \mathrm{mL}$ vs $2434.2 \pm 2305.1 \mathrm{pg} / \mathrm{mL}, P<.05$, Table 4$)$. Figure $2, B$, illustrates the efficacy of the different ultrafiltration strategies and filter types in removing IL-6, as demonstrated by the ratio of the ultrafiltrate to plasma concentrations. IL-6 was significantly better eliminated in group C than in groups $\mathrm{B}(0.02 \pm 0.017$ vs $0.005 \pm 0.009, P<.02)$ and $\mathrm{D}(0.02 \pm 0.017$ vs $0.003 \pm 0.003, P<.01)$, and there was more effective elimination of IL-6 by CUF than MUF $(0.018 \pm 0.017$ vs $0.004 \pm 0.007, P<.005)$. Ultrafiltration was more effective in removing TNF than the other mediators, with much higher mean ultrafiltrate/plasma ratios $(1.01 \pm 0.94,2.22 \pm 2.27,2.72 \pm 2.75$, and $2.01 \pm 2.34$ for groups $\mathrm{A}, \mathrm{B}, \mathrm{C}$, and $\mathrm{D}$, respectively) without differences between the groups.

\section{Clinical Outcome}

Clinical outcomes were similar in all groups and not influenced by type of filter or ultrafiltration strategy. Death (1 patient in group C), multiorgan failure (1 patient in group C), arrhythmias (4 patients in group B, and 2 in groups $\mathrm{C}$ and $\mathrm{D}$ ), infections (1 patient in groups $\mathrm{A}$ and $\mathrm{D}$, and 2 in groups $\mathrm{B}$ and $\mathrm{C}$ ), renal failure requiring temporary dialysis (1 patient in group C), revisions for bleeding ( 2 patients in group C), and duration of postoperative ventilatory support (group A $43.2 \pm 2.1$ hours, group B $55.6 \pm 57.5$ hours, 

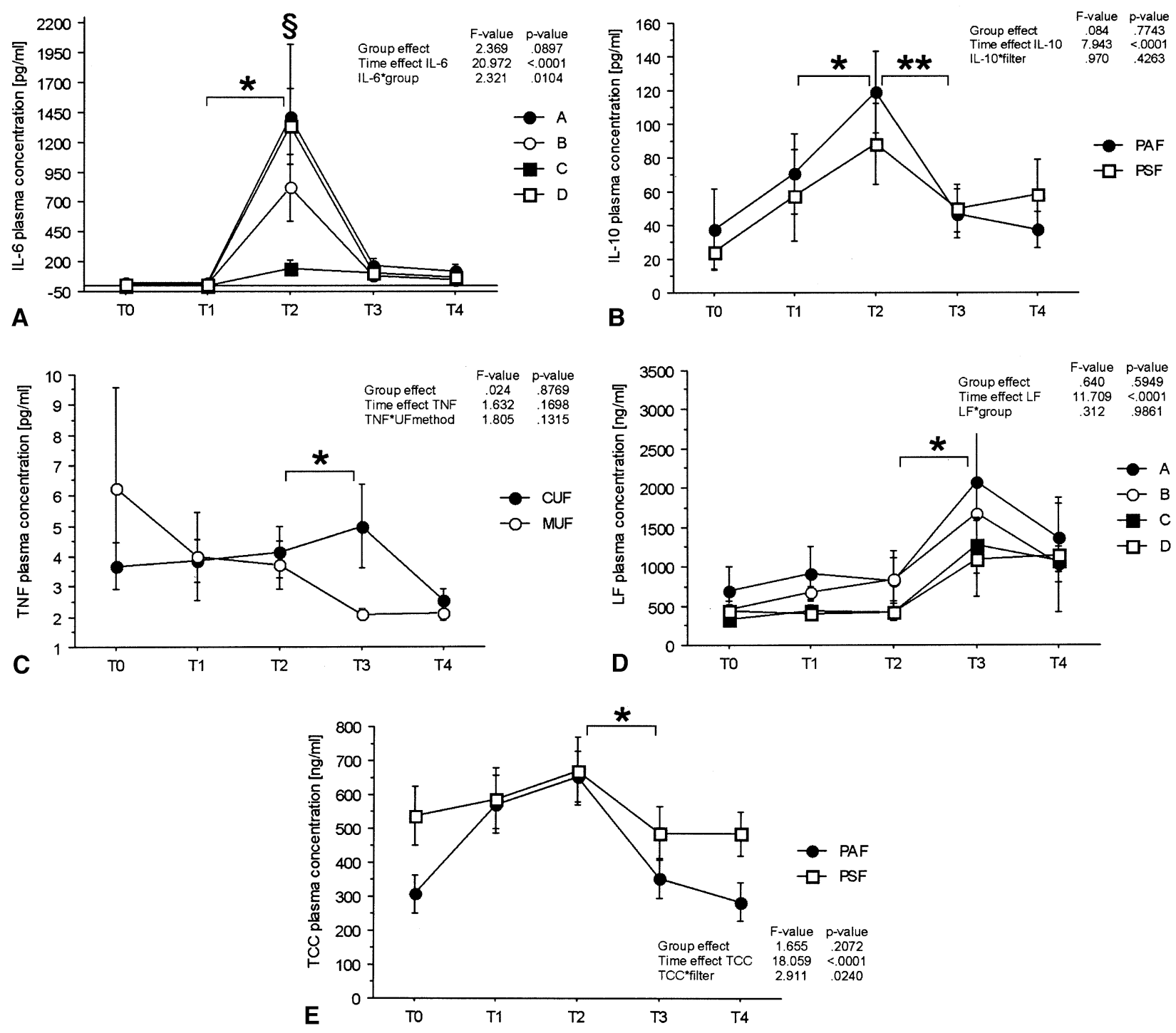

Figure 1. Repeated-measures analysis of variance, $P$ values for group effect, time effect, and interaction are depicted. A, Plasma concentrations of IL-6, stratified by groups A, B, C, and D. Asterisk indicates $P<.005$ for groups $A$ and $B$; section mark indicates $P<.01$ for group $C$ versus group $D$. B, Plasma concentrations of IL-10, stratified by filter types PAF and PSF. Asterisk indicates $P=.0002$; double asterisk indicates $P<.001$ for PAF. C, Plasma concentrations of TNF, stratified by ultrafiltration methods CUF and MUF. Asterisk indicates $P<.002$ for MUF. D, Plasma concentrations of TCC, stratified by filter types PAF and PSF. Asterisk indicates $P<.0005$ for PAF. E, Plasma concentrations of lactoferrin (LF), stratified by groups A, B, C, and D. Asterisk indicates $P<.005$ for $A$.

group C $61.9 \pm 46$ hours, group D $80.5 \pm 110.2$ hours) were all not significantly different among the groups. However, low cardiac output was significantly more frequent after the use of PSF than PAF filters $(P<.02)$. Blood losses were similar in all groups (Table 1), irrespective of filter type or ultrafiltration method used. Intraoperative red blood cell transfusion volume $(92 \pm 81 \mathrm{~mL} / \mathrm{kg}$ vs $120 \pm 62$ $\mathrm{mL} / \mathrm{kg}, P<.05)$ and total fresh-frozen plasma transfusion volume $(23 \pm 27 \mathrm{~mL} / \mathrm{kg}$ vs $40 \pm 35 \mathrm{~mL} / \mathrm{kg}, P<.05)$ were lower with the use of PAF than PSF, whereas the intraop- erative fresh-frozen plasma requirement was significantly lower with CUF than MUF $(15 \pm 21 \mathrm{~mL} / \mathrm{kg}$ vs $31 \pm 32$ $\mathrm{mL} / \mathrm{kg}, P<.05)$. Neither type of filter nor ultrafiltration method influenced intensive care unit or hospital stay. However, group D patients had significantly longer intensive care unit stays than did group A patients $(9 \pm 7.4$ days vs $4.8 \pm 2.1$ days, $P<.05$ ) and longer hospital stays than both group A patients $(21.8 \pm 19.9$ days vs $21.8 \pm 19.9$ days, $P$ $<.05)$ and group $\mathrm{C}$ patients $(21.8 \pm 19.9$ days vs $11.5 \pm$ 3.9 days, $P<.05$ ). 
TABLE 2. Plasma concentrations of lactoferrin and TCC according to ultrafiltration method or filter type

\begin{tabular}{|c|c|c|c|c|}
\hline & CUF & MUF & PAF & PSF \\
\hline \multicolumn{5}{|c|}{ Lactoferrin } \\
\hline TO & $495.1 \pm 669.3$ & $421.8 \pm 288.3$ & $529.8 \pm 662.4$ & $370.3 \pm 192.9$ \\
\hline $\mathrm{T} 1$ & $632.4 \pm 727.6$ & $544.3 \pm 255.1$ & $714.8 \pm 706.9$ & $444.3 \pm 192.2$ \\
\hline $\mathrm{T} 2$ & $578.2 \pm 792.9$ & $626.7 \pm 669$ & $718.3 \pm 957.1$ & $458.2 \pm 209.9$ \\
\hline T3 & $1531.3 \pm 2023.7^{*}$ & $1463.7 \pm 1064.1$ & $1743.9 \pm 2014.3 \dagger$ & $1215.6 \pm 1010 \ddagger$ \\
\hline $\mathrm{T} 4$ & $1159.6 \pm 1023.4$ & $1012.7 \pm 1121.2$ & $1169.7 \pm 995.5$ & $1001.5 \pm 1147.3$ \\
\hline \multicolumn{5}{|l|}{ TCC } \\
\hline TO & $415.2 \pm 346.8$ & $417.7 \pm 294.7$ & $344.7 \pm 261.4$ & $505.1 \pm 363.9$ \\
\hline $\mathrm{T} 1$ & $533.2 \pm 333.9$ & $654.4 \pm 380.6$ & $616.2 \pm 364.7$ & $564.7 \pm 358.8$ \\
\hline $\mathrm{T} 2$ & $644.3 \pm 360$ & $650.3 \pm 360.9$ & $673.1 \pm 308.3$ & $615.4 \pm 413.7$ \\
\hline $\mathrm{T} 3$ & $378.5 \pm 301.1 \ddagger$ & $502 \pm 274.8$ & $398.7 \pm 283.3 \dagger$ & $479.7 \pm 303.2$ \\
\hline $\mathrm{T} 4$ & $375.9 \pm 274.6$ & $388.8 \pm 272.4$ & $302.9 \pm 254.9$ & $465.7 \pm 266.3$ \\
\hline
\end{tabular}

All values are mean $\pm S D$.

${ }^{*} P<.0001$ for within-group difference from previous time point.

$\dagger P<.0005$ for within-group difference from previous time point.

$\ddagger P<.005$ for within-group difference from previous time point.

TABLE 3. Plasma concentrations of IL-6, IL-10, and TNF according to ultrafiltration method or filter type

\begin{tabular}{|c|c|c|c|c|}
\hline & CUF & MUF & PAF & PSF \\
\hline \multicolumn{5}{|l|}{ IL-6 } \\
\hline T0 & $13.7 \pm 38.1$ & $5 \pm 5.4$ & $13.3 \pm 36.2$ & $4.4 \pm 5.2$ \\
\hline T1 & $10.3 \pm 21.3$ & $6.4 \pm 7.1$ & $10.4 \pm 20$ & $6.1 \pm 9.4$ \\
\hline $\mathrm{T} 2$ & $773.7 \pm 1370.7^{*} \dagger$ & $1083.4 \pm 893.4 \dagger$ & $981.6 \pm 1344.6 \dagger$ & $846.6 \pm 931.7 \ddagger$ \\
\hline T3 & $125.7 \pm 111$ & $91 \pm 62.4 \ddagger$ & $120.9 \pm 115.1 \ddagger$ & $96.4 \pm 56.6 \S$ \\
\hline $\mathrm{T} 4$ & $84 \pm 136.3$ & $50.8 \pm 31.2$ & $76.6 \pm 133.4$ & $58.5 \pm 47$ \\
\hline \multicolumn{5}{|l|}{ IL-10 } \\
\hline T0 & $12.7 \pm 15.4$ & $46.2 \pm 102.1$ & $33.3 \pm 93.2$ & $24.6 \pm 41.9$ \\
\hline T1 & $48.1 \pm 95.4$ & $68.4 \pm 95$ & $59.6 \pm 92.7$ & $56.1 \pm 99.2$ \\
\hline $\mathrm{T} 2$ & $101.8 \pm 108 \|$ & $108.4 \pm 100.7 \S$ & $107.2 \pm 95.9 \ddagger$ & $102.1 \pm 114.5$ \\
\hline T3 & $40.3 \pm 43.3$ & $51.6 \pm 67.5$ & $40.8 \pm 59.3 \|$ & $51.1 \pm 52.4$ \\
\hline $\mathrm{T} 4$ & $51.4 \pm 79.4$ & $35.7 \pm 43.4$ & $35.9 \pm 44.4$ & $51.2 \pm 78.8$ \\
\hline \multicolumn{5}{|l|}{ TNF } \\
\hline T0 & $3.6 \pm 3.2$ & $5.8 \pm 13$ & $3.5 \pm 3$ & $6.3 \pm 13.8$ \\
\hline T1 & $5 \pm 6.5$ & $4.7 \pm 6.8$ & $4.8 \pm 6.3$ & $4.9 \pm 7$ \\
\hline $\mathrm{T} 2$ & $4.1 \pm 3.3$ & $4.4 \pm 4.7$ & $3.7 \pm 3$ & $4.9 \pm 5$ \\
\hline T3 & $4.6 \pm 5.5$ & $2.7 \pm 2.7 \S$ & $4.2 \pm 5.2$ & $3.1 \pm 3.3$ \\
\hline $\mathrm{T} 4$ & $2.5 \pm 1.5$ & $2.2 \pm 1$ & $2.5 \pm 1.6$ & $2.2 \pm .9$ \\
\hline
\end{tabular}

All values are mean $\pm S D$.

${ }^{*} P<.05$ for between-group difference at the same time point for CUF versus MUF.

$\dagger P<.0001$ for within-group difference from previous time point.

$\ddagger P<.0005$ for within-group difference from previous time point.

$\S P<.005$ for within-group difference from previous time point.

$\| P<.001$ for within-group difference from previous time point.

\section{Discussion}

Ultrafiltration techniques have been shown to improve postoperative hemodynamics ${ }^{9,16,17}$ and recovery of myocardial, ${ }^{18}$ cerebral, ${ }^{19}$ respiratory, ${ }^{6,11}$ and hemostatic functions ${ }^{3,9}$ after pediatric cardiac surgery, mainly because of their ability to eliminate excess free plasma water. ${ }^{3,9,10}$ Controversy exists regarding their potential to remove proinflammatory mediators (cytokines and complement factors) that play a major role in the inflammatory response to $\mathrm{CPB}^{1,2}$ and in postoperative morbidity of neonates and infants. ${ }^{9}$ Inconsistent results among studies published in the literature ${ }^{4,5,15}$ are partially explained by different ultrafiltration methods with various end points and different filter types with specific functional and physical properties. ${ }^{12}$ We wanted to analyze the influence of different filter types on the elimination of inflammatory cytokines and define the optimal combination of an ultrafiltration method with a specific filter type. Such a combination should ultimately allow more efficient elimination of cytokines.

Overall, there was little difference between the two ultrafiltration methods concerning their efficacy to remove 
TABLE 4. Concentrations of inflammatory mediators in the ultrafiltrate and amounts removed

\begin{tabular}{|c|c|c|c|c|c|}
\hline & A & B & C & $\mathbf{D}$ & $P$ value \\
\hline IL-6 in ultrafiltrate $(\mathrm{pg} / \mathrm{mL})$ & $1.28 \pm .595$ & $1.6 \pm .92$ & $1.0 \pm .02$ & $4.6 \pm 7.08$ & .39 \\
\hline IL-6 removed $(\mathrm{pg})$ & $1048.3 \pm 584$ & $1100.4 \pm 593$ & $752.1 \pm 265$ & $3247.3 \pm 5111$ & .79 \\
\hline IL-10 in ultrafiltrate $(\mathrm{pg} / \mathrm{mL})$ & $<3.0$ & $10.5 \pm 25.04$ & $<3.0$ & $<3.0$ & .98 \\
\hline IL-10 removed $(p g)$ & $2505 \pm 886$ & $10,567.6 \pm 27894$ & $2256 \pm 797$ & $2066.7 \pm 495$ & .61 \\
\hline TNF in ultrafiltrate $(\mathrm{pg} / \mathrm{mL})$ & $2.83 \pm 1.83$ & $5.22 \pm 4.67$ & $6.57 \pm 5.88$ & $7.06 \pm 4.39$ & $A: D<.02$ \\
\hline TNF removed (pg) & $2434.1 \pm 2305$ & $4216.2 \pm 4406$ & $5107.2 \pm 4864$ & $4750 \pm 2594$ & $A: D<.05$ \\
\hline TCC in ultrafiltrate $(\mathrm{ng} / \mathrm{mL})$ & $62.25 \pm 194$ & 0 & $6.75 \pm 21$ & $79.66 \pm 239$ & .77 \\
\hline TCC removed (ng) & $73,311.6 \pm 233,174$ & 0 & $6075.9 \pm 19,214$ & $54,166.9 \pm 162,501$ & .76 \\
\hline Lactoferrin in ultrafiltrate $(\mathrm{ng} / \mathrm{mL})$ & $<1$ & $<1$ & $<1$ & $<1$ & .99 \\
\hline Lactoferrin removed (ng) & $813.6 \pm 289$ & $754.5 \pm 263$ & $752 \pm 266$ & $688.9 \pm 165$ & .64 \\
\hline
\end{tabular}

All values not below level of detection are mean \pm SD.

inflammatory mediators from the blood. We found that the ultrafiltration method itself was responsible for a measurable difference for IL-6, TNF, TCC, and lactoferrin. IL-6 plasma levels were significantly lower at the end of CUF than both after MUF and after CUF with PSF. Accordingly, the ultrafiltrate/plasma ratio of IL-6 was significantly higher in the CUF group C than in the MUF groups B and D and was higher with CUF than with MUF, indicating better elimination of IL-6 with CUF than with MUF. Thus, IL-6 was removed to a significantly higher degree from the blood with CUF than with MUF, especially when combined with a PSF filter type, although no relevant differences in total amount of IL-6 filtered were found among the groups. These findings are similar to those of Wang and coworkers, ${ }^{15}$ who also found lower plasma IL- 6 concentrations after CUF than MUF with PSF but without a higher ultrafiltrate/plasma ratio of IL-6. They also found TNF to be more efficiently eliminated with MUF. Theoretically it would be more appealing to eliminate the inflammatory mediators by CUF during rewarming, the moment of their maximal release., 5 In accordance with Journois and coworkers, who found IL-6 and TNF significantly lowered with CUF and PSF in their first study ${ }^{6}$ and IL-6, TNF, IL-10, and C3a better eliminated with combined zero-balanced CUF and MUF than MUF alone with a polynitril filter in their second study, ${ }^{4}$ our findings suggest no uniform advantage of one ultrafiltration method with respect to the other concerning cytokine removal. Nevertheless, elimination of IL-6 may be of prognostic importance, because Hauser and coworkers ${ }^{21}$ showed that elevated IL-6 plasma levels 2 hours after CPB correlated significantly with postoperative morbidity and mortality. Although TCC was lower after CUF and lactoferrin was lower after MUF, both their concentrations in the ultrafiltrate were extremely low, suggesting poor filtration. Even though filtered quantities of proinflammatory markers have mostly been small in other studies as well, ${ }^{5,13,15}$ ultrafiltration is able to reduce their plasma concentrations after the operation and exert a modifying effect on the inflammatory response to $\mathrm{CPB} .^{9,15,22,23}$
Analysis of ultrafiltration efficacy in the literature has focused mainly on different ultrafiltration methods. But these various ultrafiltration methods may exert only a modest influence on cytokine elimination, which instead shows significant correlation with duration of filtration, ${ }^{6}$ and on clinical outcome, which depends more on the amount of fluid filtered than the ultrafiltration strategy being used. ${ }^{14}$ Instead, efficacy of ultrafiltration may depend on the properties of the filter type in use, which may differ considerably in their performance of solute removal and clearance of molecules. These differences are at least in part explained by the physical characteristics of the membrane used (pore diameter and electrostatic surface), which may inhibit cytokines to be passively filtered together with plasma water along a pressure gradient across the semipermeable membrane. Generally, smaller molecules such as IL-6, $, 13,15 \mathrm{IL}-$ $8,{ }^{9,15} \mathrm{TNF},{ }^{6,13,15} \mathrm{C} 3 \mathrm{a}$ or $\mathrm{C} 5 \mathrm{a},{ }^{4-6}$ and endothelin $1^{11,17}$ have been shown to be filtered with ultrafiltration. However, the efficacy of removal of different molecules from the circulation by ultrafiltration may be explained not only by their molecular weights but also by different molecular conformations and formation of complexes with physiologic soluble inhibitors. ${ }^{14}$ Furthermore, with ultrafiltration substances are not necessarily removed from the plasma only by convection. Barrera and coworkers ${ }^{24}$ showed that cytokine binding to the membrane may be an additional mechanism, which could also explain the paradoxic lack of correlation between cytokine reduction and ultrafiltrate volume found by others. ${ }^{6}$ The two filter membranes we used demonstrate some differences in their ability to eliminate cytokines. PAF was more effective lowering IL-10 and TCC plasma levels, whereas PSF was more effective lowering IL-6 and showed superior filtration quantities of TNF in the ultrafiltrate. However, filtration of IL-10, TCC, and lactoferrin was uniformly poor, as suggested by their low concentrations in the ultrafiltrate and their low ultrafiltrate/ plasma ratio. Other studies with different filters and ultrafiltration methods have also found elimination of these inflammatory markers difficult, and it has been hypothe- 
sized that their larger molecule weights inhibit efficient filtration because their levels in the ultrafiltrate have been uniformly low in most studies, ${ }^{6,9,13,15}$ irrespective of the ultrafiltration method used. TNF was the only cytokine that was filtered to a larger extent, as shown by the much higher ultrafiltrate/plasma ratios and the large amounts of TNF in the ultrafiltrate. TNF was significantly better filtered with PSF than PAF, with significant differences in its concentration in the ultrafiltrate. TNF has been found to be effectively filtered with ultrafiltration by others as well, ${ }^{6,13,15}$ with PSF $^{6,15,25}$ but also with other filter types, such as polynitril. ${ }^{4,17}$ Although filtered in larger quantities with PSF, however, plasma TNF levels did not significantly differ from those measured with PAF. This finding shows that the PSF may not be superior to the PAF to such a degree as to produce measurable differences in plasma TNF levels. Others have used PSF ${ }^{6,15,25}$ and $\mathrm{PAF}^{6,13,15}$ as well, and also different filters such as polynitril, ${ }^{4,17}$ but no one has compared one filter with another directly (eg, PSF vs PAF filters). Their results are quite comparable with ours concerning performance of individual filter types. Our comparison, however, shows a slightly superior profile of inflammatory mediator filtration of PSF compared to PAF filters, with better elimination of proinflammatory mediators such as TNF while anti-inflammatory mediators such as IL-10 are being withheld in the circulation, because a positive effect of ultrafiltration by elimination of cytokines depends on the balance between proinflammatory and anti-inflammatory cytokine levels after ultrafiltration. ${ }^{22}$

Although the difference was not statistically significant, ultrafiltration volume was smaller with MUF than with CUF in our study, in contrast to the findings of others. ${ }^{3,9}$ This reflects our practice of prolonging CUF for the whole phase of rewarming to maximize its potential benefits concerning water and cytokine elimination. With MUF, however, duration of filtration was limited to one to two cycles of the total circulating blood volume passing across the filter to keep the potentially hemodynamically unstable phase of filtration as short as possible. MUF was therefore limited to 20 minutes. Furthermore, with MUF the total volume accessible to filtration consisted of only the patient volume and was therefore much smaller than with CUF, during which patient plus CPB volume was being filtered. Nevertheless, with both methods it was always possible to remove at least the whole priming volume and reach excellent hemoconcentration, as demonstrated by an important rise in hematocrit in all groups.

Overall, our results seem to have been more influenced by the filter type used than by the ultrafiltration method applied, although differences were small. This finding puts the ongoing discussion on which ultrafiltration method may be the most effective into a new perspective and emphasizes the importance of the choice of the filter type and the need
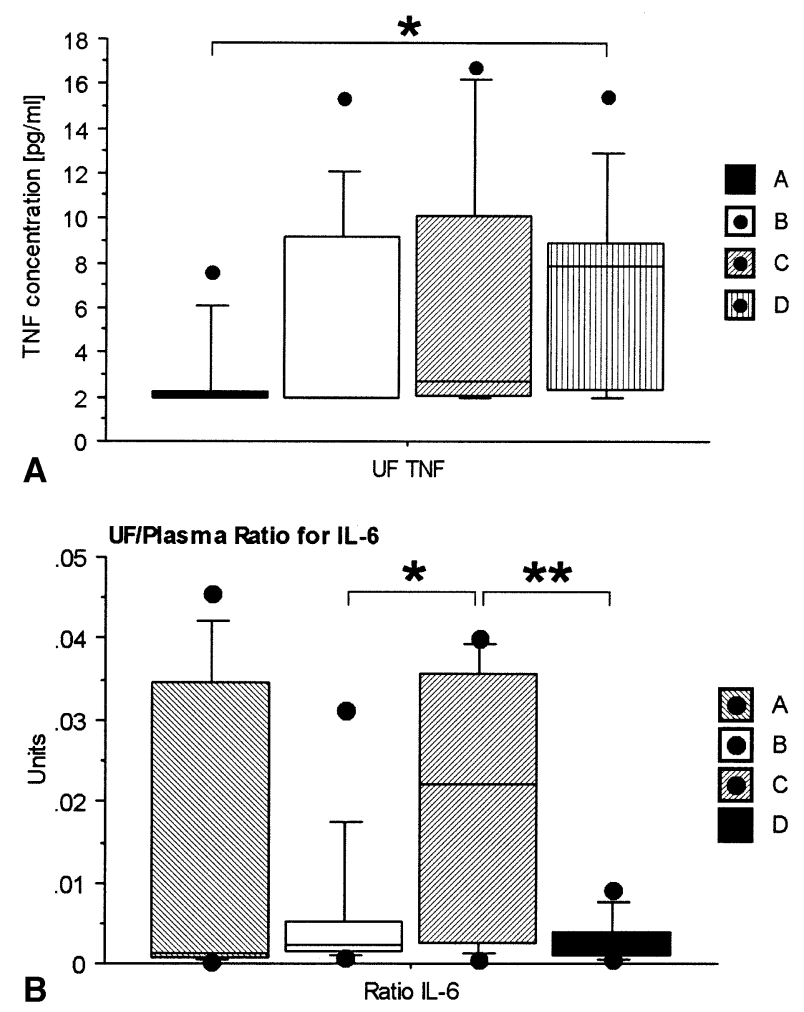

Figure 2. A, Ultrafiltrate (UF) concentrations of TNF in groups $A$, B, C, and D. Asterisk indicates $P<.02$. B, Ultrafiltrate/plasma ratio of IL-6 in groups $A, B, C$, and D. Asterisk indicates $P<.02$; double asterisk indicates $P<.01$.

for taking it into consideration during the interpretation of study results. The power of this study may be limited by the small groups, although other authors have also studied effects of ultrafiltration with groups of comparable size. ${ }^{4-6,8,11,13,15,18,19} \mathrm{We}$ decided to limit testing to the two filter types and ultrafiltration methods routinely in use at our institution. The presented results may not be directly comparable with those of others because of differences in the methods of ultrafiltration performed in this study. In particular, MUF was not performed exactly the same way as proposed by Elliott and associates. ${ }^{9}$ Although our method of MUF may not allow salvage of volume from the bypass circuit and may therefore require greater transfusion volumes of blood product, we do not think that these differences influence filter performance or cytokine elimination. The study may be further limited in not having measured the remaining potentially important inflammatory mediators, such as C3a, C5a, IL-1RA, and IL-8, or the activation of leukocytes by the granulocyte surface marker CD177, which might have shown somewhat different results. However, we chose the most important mediators from the interleukin, complement, and leukocyte systems with known effects on the inflammatory response and clinical 
parameters. There is to our knowledge currently no other study available that has directly compared different ultrafiltration methods and filter types in a prospectively randomized fashion. This study demonstrates that the contribution of the filter type to cytokine elimination obtained by ultrafiltration is not negligible and should be taken into consideration when interpreting study results.

\section{Conclusions}

In summary, the CUF with PSF combination (group C) was found to best eliminate IL-6, with no significant increase during filtration, the lowest IL-6 plasma concentrations directly after filtration, and the highest ultrafiltrate/plasma ratio. Concerning IL-10, PSF, perhaps combined with CUF, may best retain IL-10. We found TNF to be best lowered after MUF, and the largest amount in the ultrafiltrate was seen after MUF with PSF (group D), suggesting that TNF is most efficiently removed by this combination. TCC was found to be best lowered after the use of PAF and showed relatively large amounts in the ultrafiltrate after CUF with PAF (group A) but also after MUF with PSF. Should elimination of TCC be desired, PAF should not be combined with MUF, since no TCC at all was found in the ultrafiltrate after MUF with PAF (group B). Concerning lactoferrin, we found it to rise most significantly after the use of CUF with PAF (group A) and to be poorly filtered with very low concentrations in the ultrafiltate of all groups. We therefore think that the combinations we chose, especially CUF with PAF, were not suitable to eliminate lactoferrin. Taken together our results suggest that PSF is advantageous for the elimination of both IL-6 (combined with CUF) and TNF (combined with MUF) with preservation of IL-10 (combined with CUF). On the other hand, one should avoid PAF combined with MUF if TCC is the target and PAF combined with CUF for lactoferrin to be efficiently removed. Therefore the combination of CUF and MUF with a PSF may currently be the most effective strategy to remove inflammatory mediators in pediatric cardiac surgery with CPB.

We thank Sonja Bisch-Knaden, PhD, from the Department of Clinical Research, and Gerhard Gillmann, from the Institute of Social and Preventive Medicine of the University of Berne, for their statistical advice.

\section{References}

1. Edmunds LH Jr. Inflammatory response to cardiopulmonary bypass. Ann Thorac Surg. 1998;66(5 Suppl):S12-6.

2. Wan S, LeClerc JL, Vincent JL. Inflammatory response to cardiopulmonary bypass: mechanisms involved and possible therapeutic strategies. Chest. 1997;112:676-92.

3. Ungerleider RM. Effects of cardiopulmonary bypass and use of modified ultrafiltration. Ann Thorac Surg. 1998;65(6 Suppl):S35-8.

4. Journois D, Israel-Biet D, Pouard P, Rolland B, Silvester W, et al. High-volume, zero-balanced hemofiltration to reduce delayed inflammatory response to cardiopulmonary bypass in children. Anesthesiology. 1996;85:965-76.
5. Andreasson S, Göthberg S, Berggren H, Bengtsson A, Eriksson E, Risberg B. Hemofiltration modifies complement activation after extracorporeal circulation in infants. Ann Thorac Surg. 1993;56:1515-7.

6. Journois D, Pouard P, Greeley WJ, Mauriat P, Vouhe P, Safran D. Hemofiltration during cardiopulmonary bypass in pediatric cardiac surgery. Effects on hemostasis, cytokines, and complement components. Anesthesiology. 1994;81:1181-9.

7. Koutlas TC, Gaynor JW, Nicolson SC, Steven JM, Wernovsky G, Spray TL. Modified ultrafiltration reduces postoperative morbidity after cavopulmonary connection. Ann Thorac Surg. 1997;64:37-43.

8. Friesen RH, Campbell DN, Clarke DR, Tornabene MA. Modified ultrafiltration attenuates dilutional coagulopathy in pediatric open heart operations. Ann Thorac Surg. 1997;64:1787-9.

9. Elliott MJ. Ultrafiltration and modified ultrafiltration in pediatric open heart operations. Ann Thorac Surg. 1993;56:1518-22.

10. Naik SK, Knight A, Elliott M. A prospective randomized study of a modified technique of ultrafiltration during pediatric open-heart surgery. Circulation. 1991;84(5 Suppl):III422-31.

11. Bando K, Vijay P, Turrentine MW, Sharp TG, Means LJ, Ensing GJ, et al. Dilutional and modified ultrafiltration reduces pulmonary hypertension after operations for congenital heart disease: a prospective randomized study. J Thorac Cardiovasc Surg. 1998;115:517-27.

12. Gaynor JW. Use of ultrafiltration during and after cardiopulmonary bypass in children. $J$ Thorac Cardiovasc Surg. 2001;122:209-11.

13. Millar AB, Armstrong L, van der Linden J, Moat N, Ekroth R, Westwick $\mathrm{J}$, et al. Cytokine production and hemofiltration in children undergoing cardiopulmonary bypass. Ann Thorac Surg. 1993;56:1499-502.

14. Thompson LD, McElhinney DB, Findlay P, Miller-Hance W, Chen MJ, Minami M, et al. A prospective randomized study comparing volume-standardized modified and conventional ultrafiltration in pediatric cardiac surgery. J Thorac Cardiovasc Surg. 2001;122:220-8.

15. Wang MJ, Chiu IS, Hsu CM, Wang CM, Lin PL, Chang CI, et al. Efficacy of ultrafiltration in removing inflammatory mediators during pediatric cardiac operations. Ann Thorac Surg. 1996;61:651-6.

16. Bando K, Turrentine MW, Vijay P, Sharp TG, Sekine Y, Lalone BJ, et al. Effect of modified ultrafiltration in high-risk patients undergoing operations for congenital heart disease. Ann Thorac Surg. 1998;66:821-8.

17. Hiramatsu T, Imai Y, Kurosawa H, Takanashi Y, Aoki M, Shinoka T, et al. Effects of dilutional and modified ultrafiltration in plasma endothelin-1 and pulmonary vascular resistance after the Fontan procedure. Ann Thorac Surg. 2002;73:861-5.

18. Davies MJ, Nguyen K, Gaynor JW, Elliott MJ. Modified ultrafiltration improves left ventricular systolic function in infants after cardiopulmonary bypass. J Thorac Cardiovasc Surg. 1998;115:361-70.

19. Skaryak LA, Kirshbom PM, DiBernardo LR, Kern FH, Greeley WJ, Ungerleider RM, et al. Modified ultrafiltration improves cerebral metabolic recovery after circulatory arrest. J Thorac Cardiovasc Surg. 1995; 109:744-52.

20. Finn A, Naik S, Klein N, Levinsky RJ, Strobel S, Elliott M. Interleukin-8 release and neutrophil degranulation after pediatric cardiopulmonary bypass. J Thorac Cardiovasc Surg. 1993;105:234-41.

21. Hauser GJ, Ben-Ari J, Colvin MP, Dalton HJ, Hertzog JH, Bearb M, et al. Interleukin-6 levels in serum and lung lavage fluid of children undergoing open heart surgery correlate with postoperative morbidity. Intensive Care Med. 1998;24:481-6.

22. Risnes I, Ueland T, Aukrust P, Lundblad R, Baksaas ST, Mollnes TE, et al. Complement activation and cytokine and chemokines release during mediastinitis. Ann Thorac Surg. 2003;75:981-5.

23. Grunenfelder J, Zund G, Schoeberlein A, Maly FE, Schurr U, Guntli $\mathrm{S}$, et al. Modified ultrafiltration lowers adhesion molecule and cytokine levels after cardiopulmonary bypass without clinical relevance in adults. Eur J Cardiothorac Surg. 2000;17:77-83.

24. Barrera P, Janssen E, Demacker P, Wetzels J, van der Meer J. Removal of interleukin-1 beta and tumor necrosis factor from human plasma by in vitro dialysis with polyacrylonitrile membrane. Lymphokine Cytokine Res. 1992;11:99-104.

25. Shimpo H, Shimamoto A, Sawamura Y, Fujinaga K, Kanemitsu S, Onoda $\mathrm{K}$, et al. Ultrafiltration of the priming blood before cardiopulmonary bypass attenuates inflammatory response and improves postoperative clinical course in pediatric patients. Shock. 2001;16(Suppl 1):51-4. 